\title{
Role of susceptibility weighted imaging in characterization of intra- cranial lesions
}

\author{
Sahdev $\mathbf{R}^{1}$, Rao A $\mathbf{A}^{2}$, Yadu $\mathbf{N}^{3}$, Shukla $\mathbf{Y}^{4}$ \\ ${ }^{1}$ Lt Col Ravinder Sahdev, Assistant Professor, ${ }^{2}$ Lt Col Akhilesh Rao, Assistant Professor, ${ }^{3}$ Dr. Neha Yadu, Resident, \\ ${ }^{4}$ Dr. Y Shukla, Resident; all authors are affiliated with Department of Radiodiagnosis \& Imaging, Command Hospital \\ (CC), Lucknow, UP, India.
}

Address for Correspondence: Lt Col Ravinder Sahdev, Email: ravinder.sahdev@gmail.com

\begin{abstract}
Background: Intrinsic differences between magnetic susceptibility of tissues can be used to generate a unique type of contrast in Magnetic Resonance Imaging (MRI), particularly to characterize tissues and improve the sensitivity for certain lesions, most notably hemorrhagic foci or calcification. Methods: A blinded retrospective review of all brain imaging studies done at our centre between March 2014 and February 2016. One of them reviewed the MR images with exclusion of SWI, while the other radiologist reviewed the entire set of images including SWI. Their findings were then compared to study the efficacy of SWI in better detection and characterization of lesions. Results: The study included 3710 patients with different brain lesions. SWI revealed positive lesions in at least 619 of these patients which were not picked up by the radiologist interpreting the conventional MRI protocol without SWI sequence. 21 patients with traumatic brain injury, 32 patients with cerebral microbleeds, 78 patients with brain tumors, 27 patients with venous malformations, 128 patients with intra-cranial calcifications, 277 patients with infarcts and 56 patients with extra-axial hemorrhage. Out of the 619 lesions, SWI played a significant role in lesion characterization in at least 198 patients which is about $5.33 \%$ of our total cohort of 3710 patients. Conclusion: SWI images are fast sequences requiring no more than a total scan time of about 3 minutes. Inclusion of this sequence as a part of brain pathology can play an important role in diagnosing different brain lesions without missing hemorrhagic lesions thus avoiding inappropriate therapeutic regimens with catastrophic results.
\end{abstract}

Keywords: Susceptibility weighted imaging, Intra-cranial lesions, Microbleeds, Extra-axial hemorrhage, Infarct, Brain Tumor.

\section{Introduction}

Amongst the many techniques for generating contrast between tissues that are available in Magnetic Resonance Imaging (MRI), those utilizing the intrinsic differences between magnetic susceptibility are particularly useful in characterizing and improving the sensitivity for certain lesions, most notably hemorrhagic foci or calcification. Haacke et al [1] described a method referred to as susceptibility-weighted imaging (SWI), in which at sufficiently long echo times (TEs), the signal from substances with different magnetic susceptibilities becomes out of phase when compared to their neighboring tissues. In SWI, the phase images

Manuscript received $10^{\text {th }}$ October 2016

Reviewed: $26^{\text {th }}$ October 2016

Author Corrected: $10^{\text {th }}$ November 2016

Accepted for Publication $22^{\text {nd }}$ November 2016 provide excellent contrast between gray and white matter as well as materials with susceptibilities that are different from surrounding tissue such as hemorrhage, calcification and blood vessels carrying deoxygenated blood.

On SWI sequence, both para- and diamagnetic substances demonstrate low signal;however the post processing scheme of SWI involves setting positive phase values (usually found in blood and CSF) to unity and negative phase values (as usually found in deoxygenated blood) to between 1 and 0 , thus creating a filtered phase map [2]. These filtered phase sequences depict differences in substance susceptibility more accurately. The paramagnetic substances, including free 
radicals, blood products, and iron, exhibit high signal, whereas diamagnetic substances such as calcium return a low signal [3].

The filtered phase mask is subsequently multiplied (between one and four times) with magnitude images to generate minimum intensity projections that will display excellent contrast [2]

Numerous neurologic disorders can potentially benefit from this method; such as trauma, vascular malformations, tumors, aging and multiple sclerosis [4].

\section{Materials and Methods}

SWI is part of our routine 'brain imaging' protocol and has been included in all brain imaging carried out at our department. Two radiologists, who were blinded to the final diagnosis as well as each other's findings, retrospectively reviewed all brain imaging studies done at our centre between March 2014 and February 2016.

One of them reviewed the MR images with exclusion of SWI, while the other radiologist reviewed the entire set of images including SWI. Their findings were then compared to study the efficacy of SWI in better detection and characterization of lesions. In addition to conventional sequences (T1-weighted, T2-weighted, FLAIR and DWI) all suspected or diagnosed cases of cerebral infarct underwent MR Angiographic studies while patients with brain tumors underwent postcontrast T1-weighted images using gadolinium based contrast agents.

Inclusion criteria: All patients undergoing brain imaging at our center.

\section{Exclusion crite ria: Nil.}

Imaging: All patients were subjected to brain MRI examination using 1.5 Tesla MR system (Magnetom Avanto Siemens Healthcare, Erlangen, Germany) with a 12-channel head-matrix coil. SWI data were collected with a 3D, fully flow-compensated GRE sequence using the following parameters: echo time $(\mathrm{TE})=40 \mathrm{~ms}$, repetition time $(\mathrm{TR})=49 \mathrm{~ms}, 72$ slices, flip angle $(\mathrm{FA})$ $=15^{\circ}$, bandwidth $(\mathrm{BW})=77 \mathrm{~Hz} / \mathrm{px}, \mathrm{FoV}=187 \times 230$, acquisition matrix $=216 \times 320$, and voxel size $=0.72 \times$ $0.72 \mathrm{~mm} \times 3 \mathrm{~mm}$; no interpolation was applied. Total acquisition time of 2:57 min.

Image and Data Analysis: We compared their findings to analyze the role of SWI in better lesion detection and characterization.

\section{Results}

A total of 3710 patients underwent brain imaging at our centre between March 2014 and February 2016. About 45.07\% $(n=1672)$ of these patients were male while females formed the other $54.93 \%(n=2038)$.

All patients underwent conventional MRI study as well as SWI sequence. SWI revealed positive lesions in at least 619 of these patients which were not picked up by the radiologist interpreting the conventional MRI protocol without SWI sequence. These patients were categorized into seven groups, for ease of demonstration of results as given in Table 1.

Table-1: Number of cases significantly affected by SWI.

\begin{tabular}{|l|c|c|}
\hline Categories & $\begin{array}{c}\text { No. of cases with positive } \\
\text { findings on SWI }\end{array}$ & $\begin{array}{c}\text { No. of diagnoses significantly } \\
\text { affected by SWI }\end{array}$ \\
\hline 1. Trauma & 21 & 9 \\
\hline 2. Chronic microbleeds & 32 & 18 \\
\hline 3. Tumors & 78 & 17 \\
\hline 4. Venous malformations & 27 & 46 \\
\hline 5. Intra-cranial calcifications & 128 & 65 \\
\hline 6. Infarcts & 277 & 34 \\
\hline 7. Extra-axial hemorrhage & 56 & 198 \\
\hline Total & 619 & 9 \\
\hline
\end{tabular}

Traumatic Brain Injury- 21 patients with history of head trauma and neurological signs and symptoms suggestive of brain insult presented to MRI with unremarkable Computed Tomography (CT) scan. 14 of these patients showed no abnormality on MRI either, however, SWI revealed positive findings in 6 of these patients that were not detected on the 
conventional series. These findings were of multiple small scattered hemorrhagic foci consistent with diffuse axonal injury (DAI). In another 3 patients SWI confirmed the presence of sub-arachnoid hemorrhage, that was suspected on FLAIR sequences.

Cerebral Microbleeds- We encountered 32 patients where SWI revealed multiple foci of 'blooming' suggestive of cerebral microbleeds, out of whom 26 patients had a positive history of hypertension. The radiological diagnosis in these patients was chronic hypertensive encephalopathy/Amyloid angiopathy with multiple microbleeds. In 18 patients, all the lesions were noted only in SWI while conventional MRI imaging and initial CT scans were either unremarkable or showed involutional brain changes and/or few lacunar infarcts. (Figure 1).
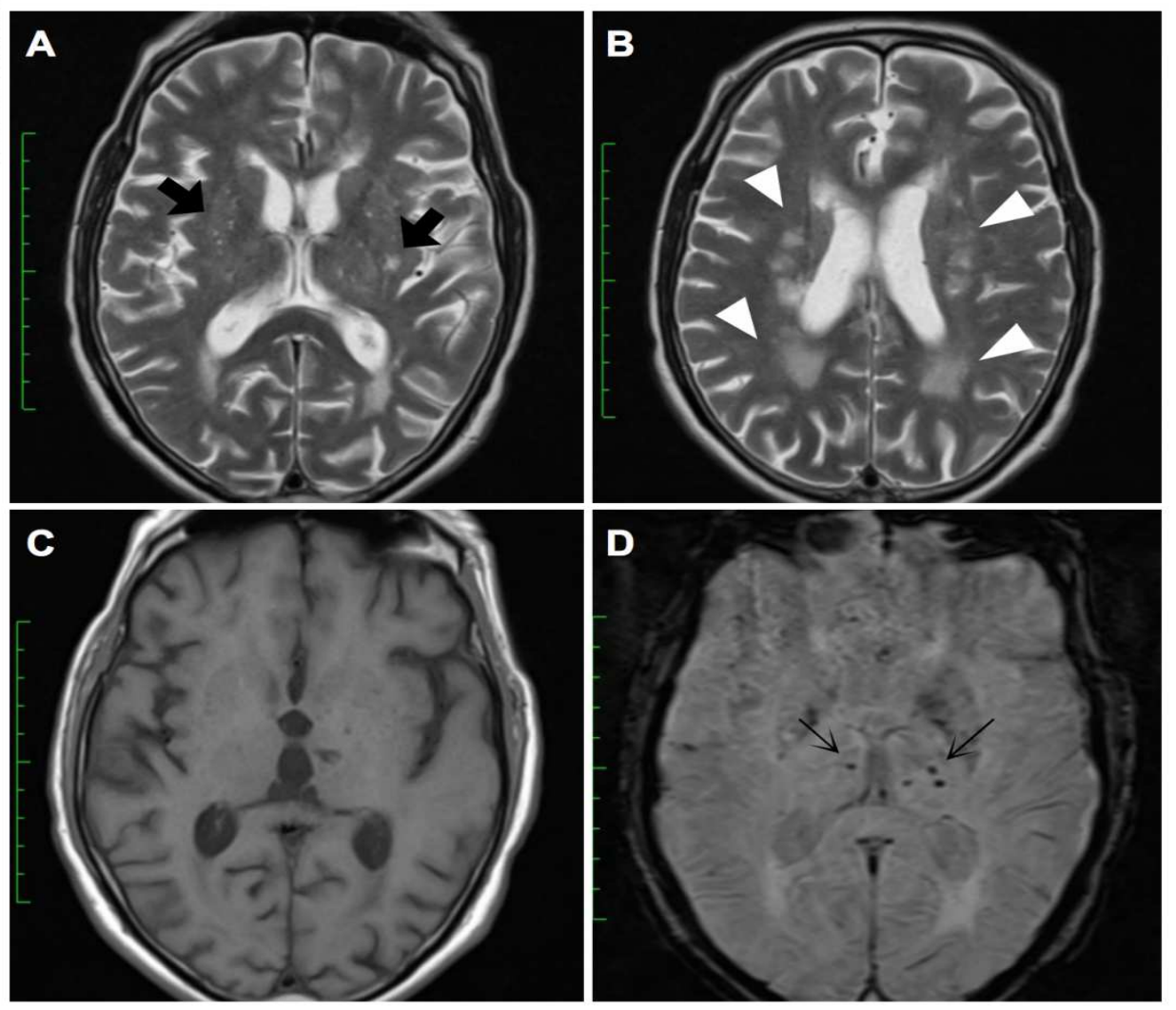

Figure 1: 63 year old hypertensive male with chronic hypertensive encephalopathy

A\&B- T2-weighted images reveal generalized prominence of the sulci, fissures and cisterns with chronic lacunar infarcts in bilateral basal ganglia (solid black arrow) and non-specific white matter changes in the periventricular region and corona radiate (white arrowheads)

C- T1-weighted images corroborate findings on T2-weighted images.

D- SWI reveals foci of magnetic susceptibility in bilateral basal ganglia thus confirming the diagnosis of chronic hypertensive encephalopathy (black arrows).

Tumors- Multiple patients $(n=78)$ with either suspicion of brain tumor or with known tumor for evaluation and grading of the tumor, underwent imaging at our centre. SWI proved to be beneficial in at least 9 patients, adding important information aiding in the diagnosis, grading and management. In 3 patients with unilateral CPA angle mass lesions, SWI revealed multiple hemorrhagic foci within the mass favoring the diagnosis of schwannoma rather than meningiomas (Figure 2). In another patient with a posterior fossa lesion, conventional imaging was unable to distinguish between intra or extra-axial location of the mass, while a displaced vessel seen between the mass and the cerebellum on SWI revealed the extra-axial origin of the tumor. In 5 patients undergoing brain imaging for follow up of glioma, SWI revealed more extensive hemorrhagic transformation within the mass denoting a higher grade of the glioma. 
Original Research Article
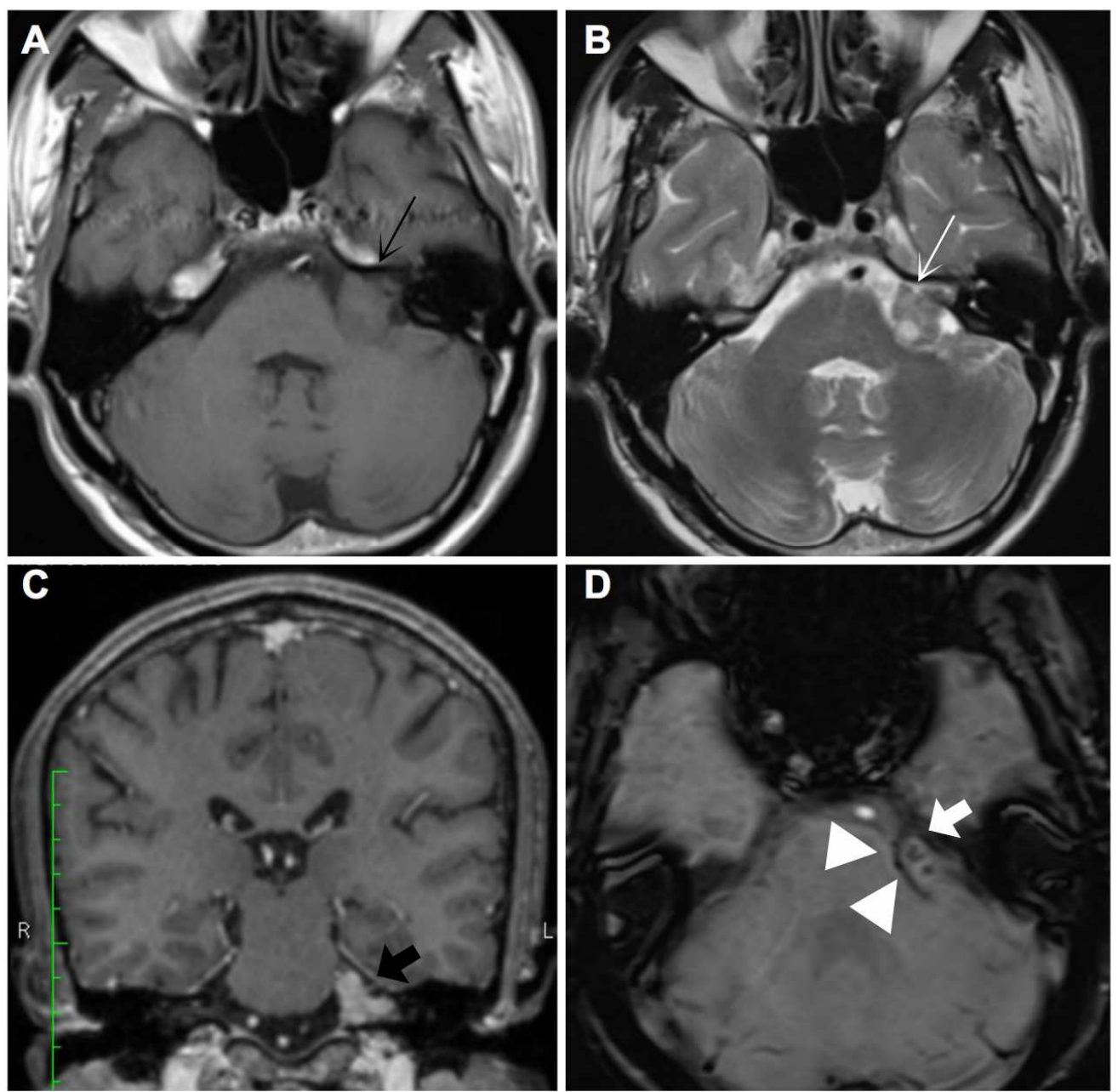

Figure 2: 39 year old male presented with left sided hearing loss
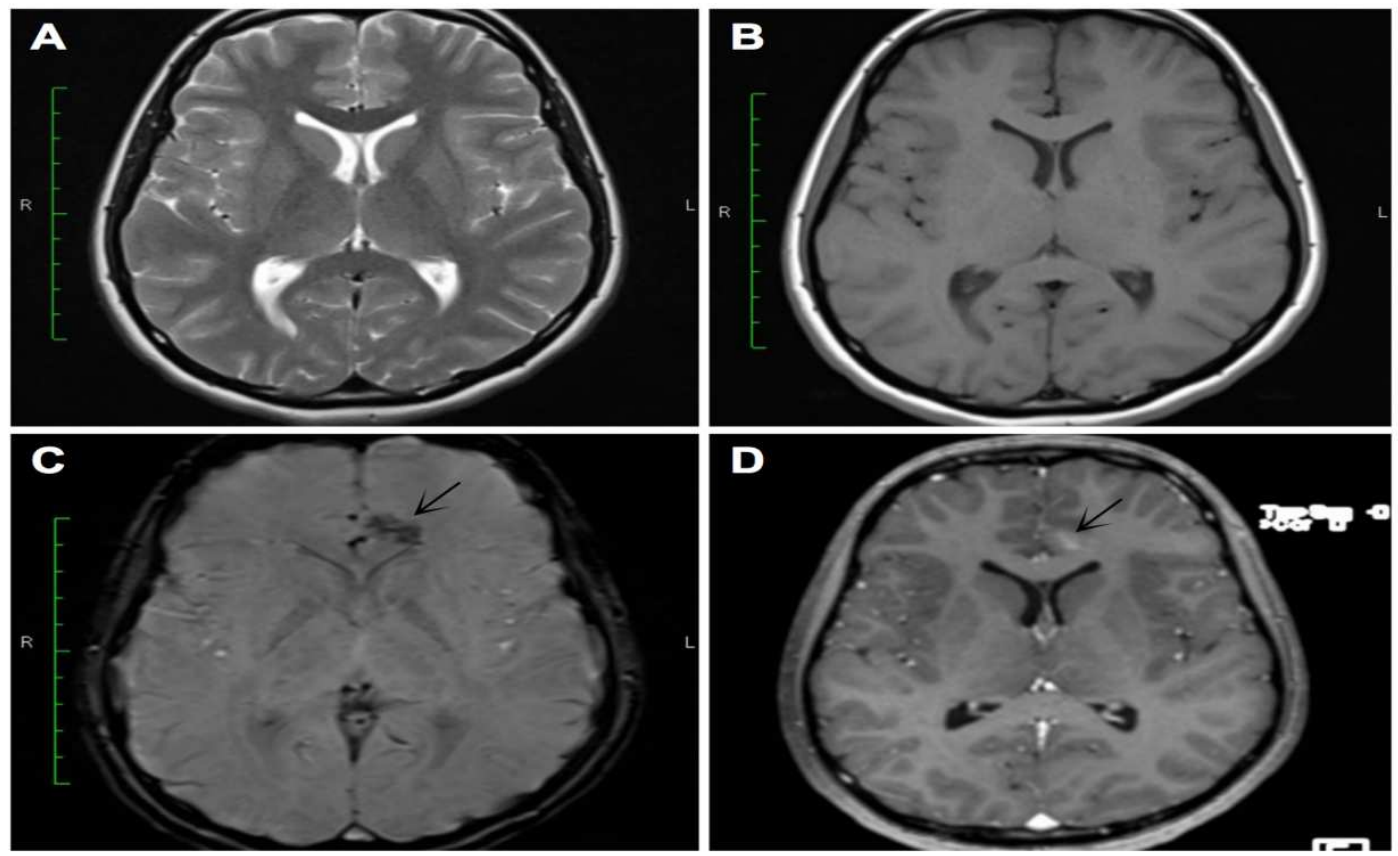

Figure 3: 16 year old female presented with headache 
A- T1-weighted images reveal an isointense extra-axial lesion in left CP angle (black arrow).

B- The lesion appears heterogeneously hyperintense on T2-weighted images (white arrow)

C- Heterogeneous post-contrast enhancement is seen in the lesion (solid black arrow).

D- SWI reveals foci of magnetic susceptibility in within the lesion, thus increasing the likelihood of the lesion being a schwannoma (solid white arrow). The extra-axial location of the tumor was further confirmed by the presence of a dural vessel between the lesion and brain parenchyma (white arrowheads).

Vascular Malformations- We encountered 27 patients with vascular malformations and venous disease, in patients undergoing MRI for neurological symptoms. In 7 patients cavernous angiomas were seen (Figure 3), 4 of these detected only on SWI images, while in another 5 patients SWI revealed capillary telangiectasias (Figure 4), which were missed on the conventional MR images. Additionally SWI was helpful in delineating the draining vessel in 5 AV malformations, venous drainage being important in the Spetzler-Martin grading scale for AV malformations with an impact on the prognostication and management of the malformation.

A \& B- T1\& T2-weighted images appear unremarkable

C- SWI reveals an ill-defined area of magnetic susceptibility in left parafalcine region.

D- T1-weighted post contrast images reveal faint brush like enhancement in the lesion suggestive of capillary telangiectasia.
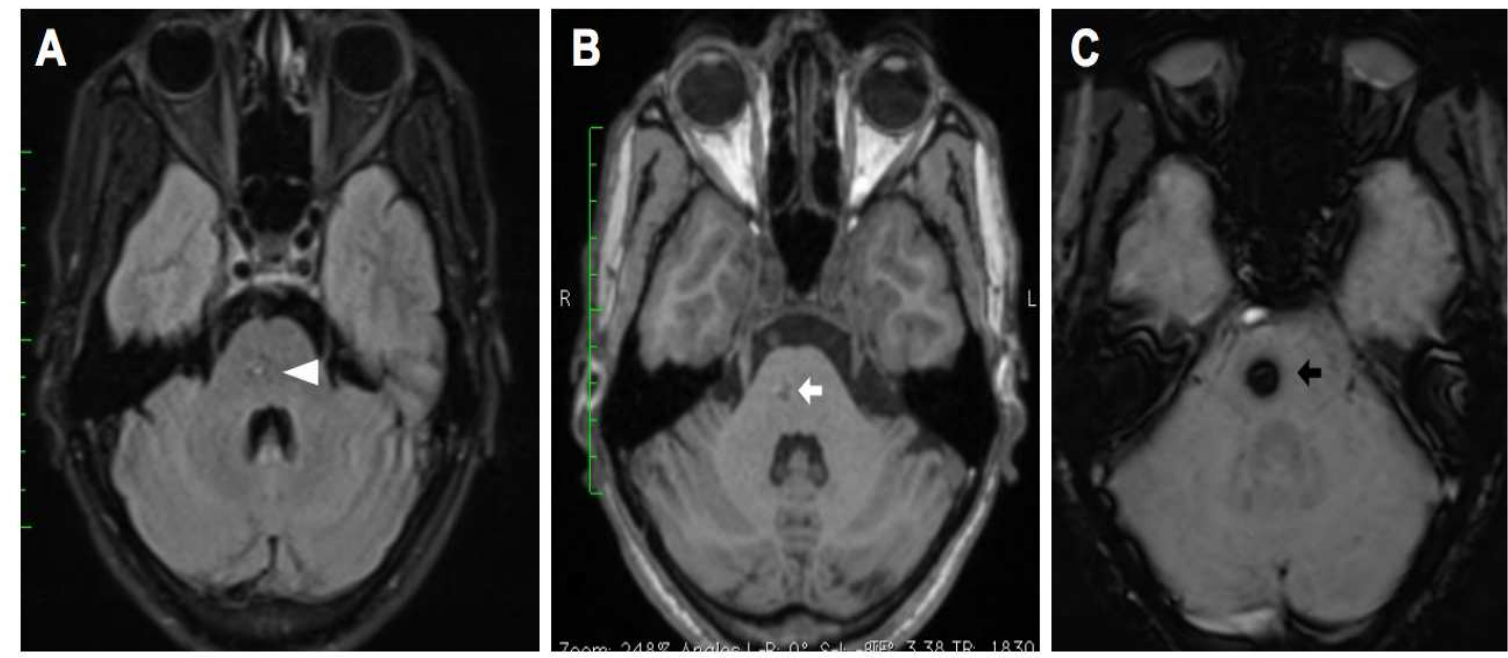

Figure 4: 33 year old male presented with history of tingling in right upper limb.

A- FLAIR images reveal an ill-defined intra-axial lesion in the pons on right side that is heterogeneously hypointense with pop-corn like hyperintense specks and a hypointense rim (white arrowhead).

B- The lesion appears heterogeneously hypointense on T1-weighted (solid white arrow).

C- Marked loss of signal is noted on SWI suggestive of cavernous angioma (solid black arrow).

Intracranial Calcifications-At least 128 patients were diagnosed with calcified granulomata and out of these SWI was useful in detecting 46 lesions that were indiscernible on other sequences. In the remaining 82 patients, low signal intensity was perceived on the conventional sequences.

Infarcts- Among the patients with findings of acute and subacute infarct on conventional sequences, 277 patients revealed positive findings on SWI. Among these, hemorrhagic transformation was detected in 189 patients on SWI as well as conventional sequences, while SWI detected hemorrhagic transformation in the form of minute hemorrhages in an additional 19 patients. Both conventional sequences and SWI were equally sensitive in detecting hematomas. SWI also detected sinus thrombosis in 69 patients. Out of these 69 patients, 46 patients were diagnosed with chronic cerebral venous sinus thrombosis on the basis of multiple collateral channels that were observed only on SWI located along an irregularly narrowed sinus on the corresponding MR Venogram images. 
Extra-axial Hemorrhage- In the last group, SWI proved to be helpful in establishing correct diagnosis in 17 patients with extra-axial collections. In 2 patients, SWI confirmed the presence of minimal intraventricular hemorrhage and in one of them the hemorrhage was seen only on SWI. In 13 patients, SWI helped confirm the diagnosis of subarachnoid hemorrhage suggested by FLAIR. In 1 patient SWI proved useful in diagnosing subdural collection of high protein content and differentiating it from subdural metastases in the form of the absence of 'blooming', while in another patient suspected of subdural metastases, SWI confirmed the diagnosis of a subdural haematoma due to presence of 'blooming'.

Finally, as we see in Table 1, out of the 619 lesions that were detected on SWI, a significant role in lesion characterization in at least 198 patients which forms about $5.33 \%$ of our total cohort of 3710 patients undergoing brain imaging.

\section{Discussion}

SWI is not a new concept, but recent advances have allowed the technique to be refined for utilization in a large number of clinical applications [5]. SWI exploits the loss of signal intensity created by various "susceptible" substances due inhomogeneity in the intrinsic magnetic field. The utility of SWI in the detection and characterization of various central nervous system lesions has been discussed under the various etiological heads below.

Traumatic Brain Injury- Detection of large amounts of intracranial hemorrhage has always been of importance when it comes to the acute surgical management of patients with traumatic brain injury. In recent times, however, identification of smaller hemorrhages and their location has gained equal importance in predicting the potential clinical outcome as well as providing useful information regarding mechanism of injury. This is particularly helpful in the evaluation of DAI, wherein small hemorrhages occur in the deeper areas of the brain that are routinely not visualized on $\mathrm{CT}$ or conventional MRI sequences. The paramagnetic properties of deoxygenated hemoglobin and other blood degradation products (like intra-cellular methemoglobin and hemosiderin) make it possible to exploit their magnetic susceptibility effects.

Scheid et al [6] compared conventional T1- and T2weighted sequences with $\mathrm{T} 2 *$-weighted GRE sequences and detected significantly more lesions in adult patients with traumatic brain injury on the GRE sequence. Tong et al [7] found SWI to detect six times the number of hemorrhagic DAI lesions compared to $\mathrm{T} 2 *$-weighted 2D GRE imaging in seven children with traumatic brain injury.

Thus, while larger hemorrhages may be easily visualized on CT and conventional MRI sequences, SWI is the only sequence available for detection of smaller hemorrhagic lesions caused by shearing forces. Also SWI has proved a useful adjunct in confirming the presence of extra-axial hemorrhage.

Cerebral Microbleeds- The role of cerebral microbleeds in vascular dementia and aging is gaining more and more attention [8]. Cerebral microbleeds are an indirect evidence of microangiopathy in patients with increased vascular vulnerability [9]. In our study, SWI proved sensitive in detecting cerebral microbleeds that were not seen in conventional MRI sequences or CT. SWI also revealed multiple foci of "blooming" predominantly in the cortical and subcortical location, thus establishing the radiological diagnosis of cerebral amyloid angiopathy. Cerebral amyloid angiopathy typically results in microhemorrhages predominantly around the arteriole vessel wall with lobar microbleeds usually related to cerebral amyloid angiopathy (largely involving the cortex and subcortical white matter within the frontal and parietal lobes), whereas microbleeds in deep white matter or the infratentorial location usually occurring as a result of hypertensive or atherosclerotic microangiopathy. In the setting of vasculitis and coagulopathies as well, SWI proves helpful in delineating the microbleeds that would otherwise have gone unnoticed if only conventional sequences were studied.

Tumors- SWI can not only increase the visibility of tumors but it also helpful in depicting hemorrhage, calcification, and increased vascularity in some neoplasms, which may reflect the tumor grade [10]. While calcification may indicate slower growth thus implying a lower grade, hemorrhage suggests rapid tumor growth and a higher tumor grade. Another application of SWI, which we have not covered in our study is the use of pre- and post-contrast SWI studies to differentiate between blood vessels and hemorrhage as the former will change in signal intensity after 
administration of contrast, whereas areas of hemorrhage will not. SWI can prove valuable in guiding the surgical or medical management of these patients.

Vascular Malformations- SWI is also superior to conventional sequences in visualizing low flow vascular malformations like telangiectasias and venous anomalies [11]. These malformations are called "occult" because they are usually not visible even on conventional angiography.

Low-flow vascular malformations are detected on conventional MR sequences only after they have bled [12]. Even post-contrast images and MR angiograms are unreliable in showing the full extent of these abnormal vessels [13].

Telangiectasias occur sporadically or may be associated with syndromes (eg, hereditary hemorrhagic telangiectasia) and are usually benign lesions. More extensive lesions may cause symptoms for which no explanation may be found with conventional sequences. SWI can successfully detect these lesions.

Cavernous angiomas that have bled previously are usually detectable on conventional imaging. However, intact lesions may be almost invisible except for illdefined blush of enhancement after contrast administration. SWI exploits the different relaxation rates between venous and arterial blood enhancing the signal-intensity loss resulting in these malformations.

Intracranial Calcifications-Discrimination between iron and calcium can be difficult even on SWI sequences, however the filtered phase images can help in differentiating amongst these two on the basis of their paramagnetic-versus-diamagnetic behaviors. Generally, deoxygenated blood/blood degradation products will appear darker depending on the resolution used and for small calcium deposits, the phase appears brighter than the background [14]. Finally, susceptibility mapping makes it possible to collapse the phase information into a susceptibility map where dark signal intensity denotes iron or deoxygenated blood/blood degradation products and bright signal intensity denotes calcium [15].

Infarcts- For purposes of revascularization in stroke, any possibility of hemorrhagic transformation is a devastating that has to be ruled out. The mechanism of this complication is a complex dynamic process that involves a combination of altered vascular permeability and microvascular injury with reperfusion integrated over time [9]. The extracellular blood degradation products are paramagnetic and thus cause local magnetic field inhomogeneity resulting in dephasing of protons. SWI being exquisitely sensitive to magnetic field inhomogeneity, can detect even very small bleeds within the infarct even those not visualized on CT [16]. SWI can also occasionally demonstrate fresh clots, that appear hypointense, within the culprit artery, accurately determining its location and proving useful in direct thrombolytic treatment [17].

Another important role of SWI in infarcts is its ability to detect even subtle prominence of medullary veins in periventricular white matter [18]. The presence of medullary vein on the side ipsilateral to the infarct is associated with hypoperfusion can be considered a predictive biomarker of poor clinical after stroke and. On the contrary, prominence of contralateral medullary veins is associated with good clinical outcome as it may indirectly reflect increased CBF and thus collateral flow on the affected side. Studies have also shown a strong correlation between prominence of ipsilateral medullary vein and hemorrhagic transformation of the infarct [19].

Extra-axial Hemorrhage- Mittal et al [17] have also found SWI sensitive in not only detecting intra-axial hemorrhages but also those occurring in the extra-axial location. In their study, SWI proved superior even to CT in demonstrating intraventricular hemorrhage and subarachnoid hemorrhage.

\section{Conclusion}

SWI images are fast sequences requiring no more than a total scan time of about three minutes. Phase images in combination with the postprocessed minimum intensity projections display excellent contrast and resolution even for small anatomic structures that may not be indistinguishable on the corresponding conventional images.

We propose that susceptibility weighted imaging be included as a routine sequence when examining any brain pathology. These sequences can play an important role in diagnosing and characterizing different brain lesions as well as increasing the sensitivity to hemorrhagic lesions. This will lead to better patient outcomes by avoiding inappropriate therapeutic regimens with catastrophic results.

Funding: Nil, Conflict of interest: None initiated, Permission from IRB: Yes 


\section{References}

1. Haacke EM, Xu Y, Cheng YC, et al. Susceptibility weighted imaging (SWI). Magn Reson Med 2004;52 (3):612-618. DOI: 10.1002/mrm.20198.

2. Akter M, Hirai T, Hiai $\mathrm{Y}$, et al. Detection of hemorrhagic hypointense foci in the brain on susceptibility-weighted imaging: clinical and phantom studies. Acad Radiol 2007;14:1011-1019.DOI: 10.1016/j.acra.2007.05.013

3. R. J. Robinson and S. Bhuta. Susceptibility-weighted imaging of the brain: current utility and potential applications. Journal of Neuroimaging, vol. 21, no. 4, pp. 189-204, 2011. doi: 10.1111/j.1552-6569.2010. 00516.x. Epub 2011 Jan 31.

4. Haacke EM, Mittal S, Wu Z, Neelavalli J, Cheng YC. Susceptibility-weighted imaging: technical aspects and clinical applications, part 1. AJNR Am J Neuroradiol. 2009 Jan;30(1):19-30. doi: 10.3174/ajnr. A1400. Epub 2008 Nov 27.

5. Tong KA, Ashwal S, Obenaus A, Nickerson JP, Kido D, Haacke EM. Susceptibility-weighted MR imaging: a review of clinical applications in children. Am J Neuroradiol 2008;29(1):9-17. http://www.ajnr.org/ content/29/1/9.long

6. Scheid R, Preul C, Gruber O, et al. Diffuse axonal injury associated with chronic traumatic brain injury: evidence from $\mathrm{T} 2 *$-weighted gradient-echo imaging at 3T. AJNR Am J Neuroradiol 2003;24:1049-56. http: //www.ajnr.org/content/24/6/1049.long

7. Tong KA, Ashwal S, Holshouser BA, et al. Diffuse axonal injury in children: clinical correlation with hemorrhagic lesions. Ann Neurol 2004;56:36-50. DOI: 10.1002/ana.20123.

8. Won Seo S, Hwa Lee B, Kim EJ, et al. Clinical significance of microbleeds in subcortical vascular dementia. Stroke. 2007;38(6):1949-1951. ttp://dx.doi. org/10.1161/STROKEAHA.106.477315

9. Nighoghossian N, Hermier M, Adeleine P, BlancLasserre K, Derex L, Honnorat J, et al. Old microbleeds are a potential risk factor for cerebral bleeding after ischemic stroke: a gradient-echo $\mathrm{T} 2 *$-weighted brain MRI study. Stroke 2002;33(3):735. http://dx.doi.org/ $10.1161 / \mathrm{hs} 0302.104615$
10. Sehgal V, Delproposto Z, Haddar D, et al. Susceptibility-weighted imaging to visualize blood products and improve tumor contrast in the study of brain masses. J Magn Reson Imaging 2006;24:41-51. https://www.ncbi.nlm.nih.gov/pubmed/16755540

11. Lee BC, Vo KD, Kido DK, et al. MR highresolution blood oxygenation level-dependent venography of occult (low-flow) vascular lesions. AJNR Am J Neuroradiol 1999;20:1239-42. PMID: 10472978

12. New PFJ, Ojeman RG, Davis KR, et al. MR and CT of occult vascular malformations of the brain. . AJNR Am J Neuroradiol 1986;7:771-779. http://www.ajnr.org /content/7/5/771 .abstract?ijkey=5dff92f1 $1 \mathrm{cfc} 2681980 \mathrm{cb}$ ec7d7d83f3a2de04e71\&keytype2=tf_ipsecsha\#cited-by

13. Marchal G, Bosmans H, Fraeyenhoven LV, Wilms G, Hecke PV, Plets C.A.L.B. Intracranial vascular lesions. Optimization and clinical evaluation of threedimensional time-of-flight MR angiography. Radiology 1990;175:443-448. DOI: http://dx.doi.org/10. 1148/ radiology.175.2.2326471.

14. Xu Y, Haacke EM. The role of voxel aspect ratio in determining apparent vascular phase behavior in susceptibility weighted imaging. Magn Reson Imaging 2006;24:155-60. DOI: 10.1016/j.mri.2005.10.030 Epub 2005 Dec 27.

15. Neelavalli J, Chen YCN, Haacke EM. Improved Fourier based Method for Calculating Field Inhomogeneity from Known Susceptibility Distribution: Proceeding of the International Society of Magnetic Resonance in Medicine, May 19-25,2007.15:1016. http://cds.ismrm.org/ismrm-2007/files/1_program.pdf.

16. Santhosh K, Kesavadas C, Thomas B, Gupta AK, Thamburaj K, Kapilamoorthy T. Susceptibility weighted imaging: a new tool in magnetic resonance imaging of stroke. Clin Radiol 2009;64(1):74-83. DOI: http://dx.doi.org/10.1016/j.crad.2008.04.022

17. Mittal S, Wu Z, Neelavalli J, Haacke EM. Susceptibility-weighted imaging: technical aspects and clinical applications. Part 2. Am J Neuroradiol 2009;30(2):232. doi: 10.3174/ajnr.A1461. Epub 2009 Jan 8. 


\section{Original Research Article}

18. Yua X, Yuanc L, Jacksond A, et al. Prominence of Medullary Veins on Susceptibility-Weighted Images Provides Prognostic Information in Patients with Subacute Stroke. AJNR 2016 37: 423-429. doi: 10.3174/ajnr.A4541. Epub 2015 Oct 29.
19. Terasawa Y, Yamamoto N, Morigaki R, et al. Brush sign on 3-T T2*-weighted MRI as a potential predictor of hemorrhagic transformation after tissue plasminogen activator therapy. Stroke 2014;45:274-76. doi:10.1161/ STROKEAHA.113.002640. Epub 2013 Oct 30.

\section{How to cite this article?}

Sahdev R, Rao A, Yadu N, Shukla Y. Role of susceptibility weighted imaging in characterization of intra-cranial lesions.Int J Med Res Rev 2016;4(11):2006-2014.doi:10.17511/ijmrr. 2016.i11.19. 